\title{
VARIABLE STIFFNESS HAND PROSTHESIS: A SYSTEMATIC REVIEW
}

\section{S. Cecilia Tapia Siles, Oscar Urquidi Gandarillas y Markus Pakleppa}

\begin{abstract}
Prosthetics is an important field in engineering due to the large number of amputees worldwide and the associated problems such as limited functionality of the state of the art. An important functionality of the human hand is its capability of adjusting the stiffness of the joints depending on the currently performed task. For the development of new technology it is important to understand the limitations of existing resources. As part of our efforts to develop a variable stiffness grasper for developing countries a systematic review was performed covering technology of body powered and myoelectric hand prosthesis. Focus of the review is readiness of prosthetic hands regarding their capability of controlling the stiffness of the end effector. Publications sourced through three different digital libraries were systematically reviewed on the basis of the PRISMA standard. We present a search strategy as well as the PRISMA assessment of the resulting records which covered 321 publications. The records were assessed and the results are presented for the ability of devices to control their joint stiffness. The review indicates that body powered prosthesis are preferred to myoelectric hands due to the reduced cost, the simplicity of use and because of their inherent ability to provide feedback to the user. Stiffness control was identified but has not been fully covered in the current state of the art. In addition we summarise the identified requirements on prosthetic hands as well as related information which can support the development of new prosthetics.
\end{abstract}

Keywords: Hand Prosthesis, Prosthetics, Variable Stiffness, Compliant Joint.

DOI: 10.23881/idupbo.017.1-8i 